Check for updates

Cite this: RSC Adv., 2017, 7, 42105

Received 8th July 2017

Accepted 24th August 2017

DOI: $10.1039 / c 7 r a 07514 f$

rsc.li/rsc-advances

\section{Enhanced efficiency and stability of inverted perovskite solar cells by interfacial engineering with alkyl bisphosphonic molecules $\uparrow$}

\author{
Nan Li, ${ }^{a}$ Changmei Cheng, ${ }^{\text {bb }}$ Hainan Wei, ${ }^{b}$ Hongbin Liu, ${ }^{c}$ Xiaosong Li, ${ }^{c}$ Wenzhe Li (D) d \\ and Liduo Wang (iD) *a
}

The moisture instability of perovskite materials especially under illumination has engendered severe hindrance toward future industrial applications for high-efficiency and stable perovskite solar cells. Here, we designed and synthesized a series of hydrophobic alkyl bisphosphonic molecules which served as interfacial layers between a perovskite and $\mathrm{PC}_{61} \mathrm{BM}$ to improve the moisture and light-stability of the inverted PVSCs. The steric arrangement of the bisphosphonic molecules suppressed the infiltration of moisture and oxygen inside the perovskite film under humidity and continuous illumination, and decreased the loss of halide and methylammonium ions as revealed by the lower $\mathrm{Pbl}_{2}$ and $\mathrm{Pb}^{0}$ in the film. When exposed to $50-60 \% \mathrm{RH}$ and continuous AM1.5G illumination, devices after undergoing interfacial treatment retained $70 \%$ of the initial power conversion efficiency, while the control device totally failed, suggesting markedly improved moisture and light-stability by the interfacial engineering. Moreover, the treated devices showed almost no degradation after being stored in an ambient atmosphere for $300 \mathrm{~h}$.

\section{Introduction}

Solution processed metal halide perovskite solar cells (PVSCs) have emerged as promising candidates in the past few years for next-generation solution-processed photovoltaics due to their potential for high power conversion efficiency (PCE), low-cost solution processing and large-area modules. ${ }^{1-4}$ Thanks to their appealing semiconductor properties including direct band gaps, long charge carrier diffusion length and low density of deep-level defects, the PCE of PVSCs has been improved from $3.8 \%$ to $22.1 \%$ in the past few years. Unfortunately, compared with their excellent photovoltaic performance, the long-term stability is a growing concern due to the rapid degradation of PVSCs upon exposure to moisture, light and thermal treatment..$^{5-9}$

Typically, degradation is acknowledged to be induced by the easy hydrolyzation and decomposition of perovskites in the presence of water and oxygen, ${ }^{10-15}$ which could be accelerated

${ }^{a}$ Key Lab of Organic Optoelectronics, Molecular Engineering of Ministry of Education, Department of Chemistry, Tsinghua University, Beijing, 100084, China. E-mail: chldwang@mail.tsinghua.edu.cn

${ }^{b}$ Key Laboratory of Bioorganic Phosphorus Chemistry and Chemical Biology, Ministry of Education, Department of Chemistry, Tsinghua University, Beijing, 100084, China. E-mail: chengcm@mail.tsinghua.edu.cn

'Department of Chemistry, University of Washington, Seattle, Washington, 98195, USA ${ }^{d}$ Institute of New Energy Technology, College of Information and Technology, Jinan University, Guangzhou 510632, China

$\dagger$ Electronic supplementary information (ESI) available. See DOI: 10.1039/c7ra07514f under light illumination. Methylammonium lead iodide $\left(\mathrm{MAPbI}_{3}\right)$ was reported to decompose into $\mathrm{PbI}_{2}, \mathrm{CH}_{3} \mathrm{NH}_{2}$ and $\mathrm{HI}$ upon exposure to ambient and light, which causes serious loss of photovoltaic performance. ${ }^{10,16}$ Interfacial engineering was reported to provide efficient protection of perovskite films from moisture and oxygen in the atmosphere ${ }^{17}$ Particularly, inorganic interlayers such as $\mathrm{SnO}_{2},{ }^{18,19} \mathrm{ZnO},{ }^{20-22} \mathrm{Zn}_{2} \mathrm{SnO}_{4}{ }^{23,24} \mathrm{BaSnO}_{3}$ (ref. 25) and $\mathrm{Ti}(\mathrm{Nb}) \mathrm{O}_{x}$ (ref. 26) have been proposed to prevent the moisture and oxygen from permeation and reaction with the perovskite layer, but the hydrothermal synthesis of the inorganic nanoparticles always required a complicated process and higher temperature. Besides, the hydrophobic molecules such as butylamine, ${ }^{27,28}$ phenethylamine ${ }^{29}$ and polyethylenimine ${ }^{30}$ could enhance the humidity stability via assembling along the surface and grain boundaries of perovskite crystals and impeding the infiltration of moisture into the inner perovskite layer. However, the higher exciton binding energy by the increased van der Waals interaction yielded low PCE around $12.52 \%$ (ref. 28) and restricted their further progress. Moreover, surface treatment with dodecyltrimethoxysilane ${ }^{31}$ and insulating aluminium oxide $^{32-36}$ have been applied to improve the moisture tolerance and air stability of perovskite films, which demonstrated a powerful strategy for fabricating stable PVSCs. Although numerous approaches have been employed to prolong the air exposure time of PVSCs, the devices still suffered from the photochemical degradation ${ }^{15,37}$ during the outdoor operation, which could accelerate the moisture-induced decomposition. The detailed investigation on the ambient stability under light 
illumination is still lacking, which is significant for fabricating robust PVSCs and promoting the practical application.

Herein, we carefully studied the moisture and light stability of $\mathrm{MAPbI}_{3}$, and rationally designed a series of low-temperature synthesized alkyl bisphosphonic molecules, which were applied as an interfacial interlayer in inverted PVSCs for fabricating high-efficiency and stable devices. The results indicated that $\mathrm{MAPbI}_{3}$ underwent severe degradation along with the generation of $\mathrm{PbI}_{2}$ and $\mathrm{Pb}^{0}$ under exposure to moisture and light. Nevertheless, by the interfacial treatment with the alkyl bisphosphonic molecules, the degradation process was effectively retarded. On the one hand, the hydroxyl and oxygen of the bisphosphonic group formed a ten-membered ring with a diameter smaller than the van der Waals radius of a single oxygen atom, successfully hindering the oxygen and moisture infiltration in perovskite films and suppressing the loss of methylammonium and iodide from the lattice. On the other hand, the bisphosphonic groups could efficiently passivate the unsaturated $\mathrm{Pb}$ during the aging process and reduce the unsatisfied recombination. Our systematical investigation demonstrated that both the photovoltaic performance and the stability were remarkably enhanced by the interfacial treatment, revealing the effectivity of interfacial treatment in moisture and light-stability improvement for highly performed inverted PVSCs.

\section{Experimental}

\section{Materials}

Nickel acetate tetrahydrate was purchased from Aladdin. Lead iodide $\left(\mathrm{PbI}_{2}\right)$ was purchased from Alfa Asear. Methylammonium iodide (MAI) and bathocuproine (BCP) were purchased from Xi'an Polymer Light Technology Corp. $\mathrm{PC}_{61} \mathrm{BM}$ was purchased from Luminescence Technology Corp. All materials were used as received. The detailed synthesis method of alkyl bisphosphonic molecules was described in the ESI. $\dagger$

\section{Device fabrication}

ITO glasses were alternately cleaned by deionized water, acetone, ethanol and isopropanol with a 15 min ultrasonic bath. $\mathrm{NiO}_{x}$ films were fabricated as reported in previous work. ${ }^{38}$ Before use, the clean substrates were treated with UV ozone for $20 \mathrm{~min}$. The $1 \mathrm{M}$ perovskite precursor solution was prepared by dissolving equimolar $\mathrm{PbI}_{2}$ and MAI in the mixed dimethyl sulfoxide (DMSO)/1,4-butyrolactone (GBL) solution with volume ratio $3: 7$. The solution was stirred at $50{ }^{\circ} \mathrm{C}$ for $1 \mathrm{~h}$. After cooled down, $180 \mu \mathrm{L}$ precursor solution was dropped on the $\mathrm{NiO}_{x}$ substrate and spin coated at $1000 \mathrm{rpm}$ for $15 \mathrm{~s}$ and $4000 \mathrm{rpm}$ for 45 s. During the second step, $500 \mu \mathrm{L}$ chlorobenzene was dropped onto the film at the last $15 \mathrm{~s}$. Subsequently, the raw films were heated at $100{ }^{\circ} \mathrm{C}$ for $10 \mathrm{~min}$. For the preparation of the saturated bisphosphonic solution, we mixed $5 \mathrm{mg}$ powders of the bisphosphonic molecules and $5 \mathrm{~mL}$ chlorobenzene together, and stirred it for $12 \mathrm{~h}$ at room temperature. A cloudy liquid was obtained due to the low solubility of the bisphosphonic molecules. Then, the cloudy liquid was filtered by a $220 \mu \mathrm{m}$ PVDF filter to get the clear saturated solution. Subsequently, the interfacial treatment was performed by spin coating the saturated solution of alkyl bisphosphonic molecules onto perovskite films at $5000 \mathrm{rpm}$ for $60 \mathrm{~s}$. The deposition method of $\mathrm{PC}_{61} \mathrm{BM}$ and $\mathrm{BCP}$ was reported before. ${ }^{39}$ Finally, $120 \mathrm{~nm}$ silver was deposited by vacuum evaporation as the electrode with a $0.16 \mathrm{~cm}^{2}$ thin mask under $10^{-4} \mathrm{~Pa}$.

\section{Characterization}

The morphologies of perovskite films were characterized by a high-resolution scanning electron microscopy (HR-SEM) (Jeol, JSM-7401F). X-ray diffraction (XRD) patterns were collected with smart lab instruments $\mathrm{CuK} \alpha$ beam $(\lambda=1.54 \AA)$. The absorption spectra were measured by a Hitachi model U-3010 UV-vis spectrophotometer. The Fourier transform infrared (FTIR) spectra were obtained by a Perkin-Elmer Spectrum GX FTIR spectrometer. X-ray photoelectron spectroscopy (XPS) was performed with a PHI 5300 ESCA Perkin Elmer spectrometer. The contact angles were tested by an optical CA meter (OCA 15pro, Dataphysics). The time-resolved photoluminescence (PL) spectra was measured by FLIM with a FV1200 laser scanning confocal microscopy. A $488 \mathrm{~nm}$ pulsed diode laser was used for excitation with repetition rates at $40 \mathrm{MHz}$. The emission was filtered through a 50/50 dichroic beam splitter and a 700$800 \mathrm{~nm}$ long pass filter. The conductive atomic force microscopy (c-AFM) was carried out by a BRUKER Dimension Icon microscope. A multi-75E-G probe (PF TUNA) was used for the detection. Perovskite films on ITO substrates were connected to the test platform with silver paste. A bias voltage of $4 \mathrm{~V}$ was applied to get an acceptable current resolution. $J-V$ curves were measured at a scan rate of $100 \mathrm{mV} \mathrm{s}^{-1}$ by a Keithley 2400 source meter under one sun illumination (AM1.5G, $100 \mathrm{~mW} \mathrm{~cm} \mathrm{~cm}^{-2}$ ), simulated by a solar simulator (ORIEL 81193) which was calibrated with an NREL-calibrated silicon solar cell. The active area of each device aperture was $0.16 \mathrm{~cm}^{2}$ and a mask of $0.09 \mathrm{~cm}^{2}$ was used during the test.

\section{Results and discussion}

Herein, we design a series of alkyl bisphosphonic molecules as illustrated Fig. 1a, nonane-1,1-diyldiphosphonic acid, tridecane1,1-diyldiphosphonic acid, pentadecane-1,1-diyldiphosphonic acid and heptadecane-1,1-diyldiphosphonic acid. The alkyl bisphosphonic molecules were chosen as the passivation molecules based on the following rationale. Firstly, bisphosphonic groups could coordinate with $\mathrm{Pb}$ and enhance the interaction with the $\mathrm{Pb}-\mathrm{I}$ framework. Secondly, the hydrophobicity of the alkyl chains could effectively block the moisture from penetrating into the devices. In addition, the bisphosphonic molecules with long alkyl chains would not break the 3D structure of the pristine perovskite and help remain the outstanding absorption and charge transport properties of the $3 \mathrm{D}$ structure.

Methylammonium lead iodide films were fabricated by the conditional anti-solvent engineering as illustrated in Fig. 1a. Afterwards, the as-synthesized alkyl bisphosphonic molecules were assembled on the perovskite films by a simple spin coating 
(a)
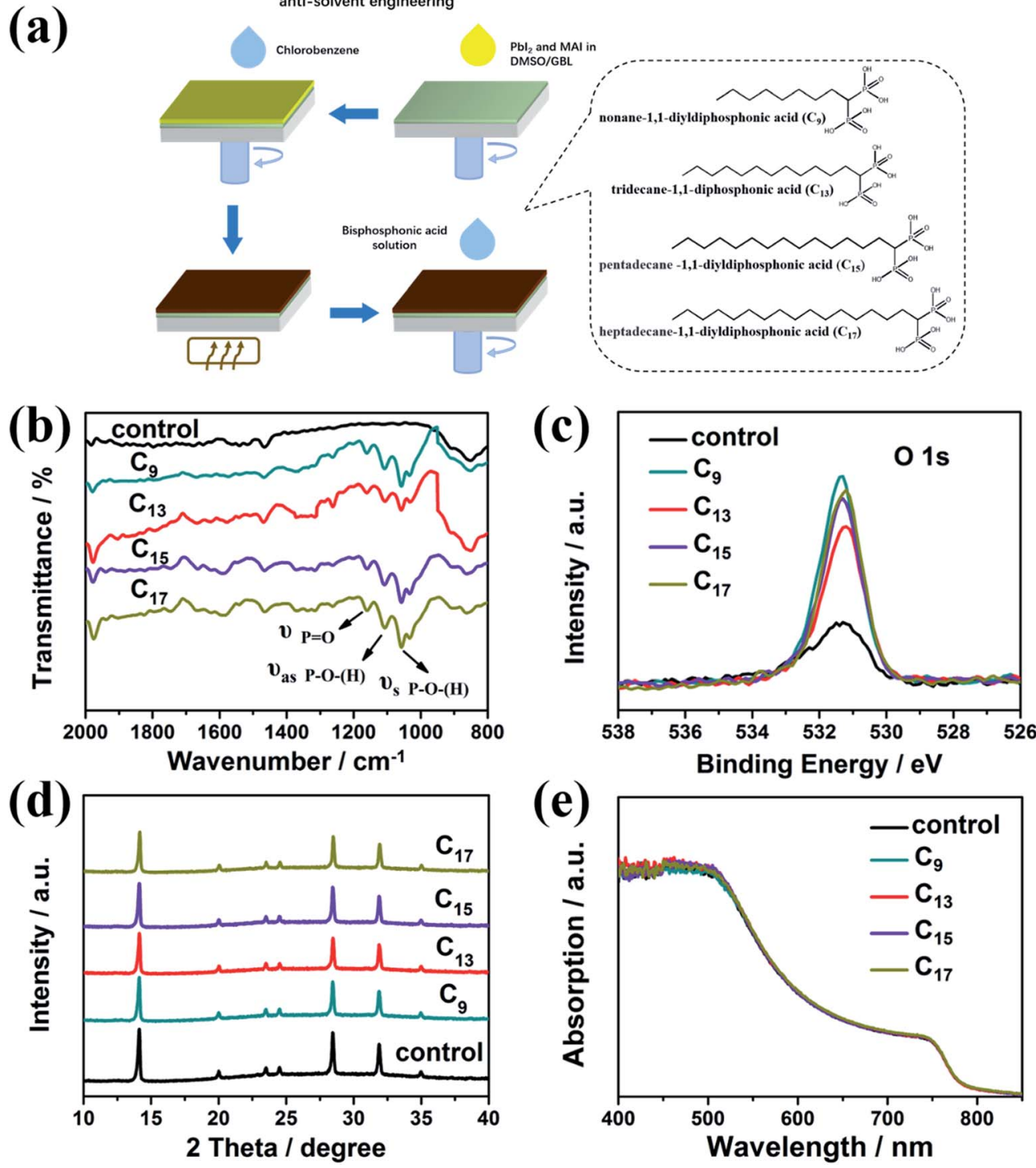

Fig. 1 (a) The scheme of the perovskite fabricating process and the interfacial treatment with the bisphosphonic acids. (b) FTIR spectra, (c) XPS spectra of O 1s, (d) XRD spectra, and (e) UV-vis spectra of the perovskite films w/o interfacial treatment.

method. Hydrophobic alkyl chains with different length were introduced to study the general roles of such hydrophobic bisphosphonic molecules, briefly labeled as $\mathrm{C}_{9}, \mathrm{C}_{13}, \mathrm{C}_{15}, \mathrm{C}_{17}$ for clarification. Firstly, FTIR spectra and XPS spectra were performed to characterize the assembled organic layer on ITO/perovskite films. All the films were kept in the nitrogen glovebox before tested in the ambient air. As shown in Fig. 1b, after the interfacial treatment, three additional absorption peaks around $1000-1200 \mathrm{~cm}^{-1}$ appeared, corresponding to the asymmetrical stretching vibration and symmetrical stretching vibration of $\mathrm{P}-\mathrm{O}$ bond, stretching vibration of $\mathrm{P}=\mathrm{O}$ bond, respectively, indicating the bisphosphonic molecules were successfully assembled on the perovskite films. The slight increase of the peak around $2000 \mathrm{~cm}^{-1}$, which was corresponding to the out-plane flexural vibration of $\mathrm{C}-\mathrm{H}$ bond in the benzene ring, was proposed to be caused by the solvent of bisphosphonic molecules, chlorobenzene. Since the XPS signal peaks of $\mathrm{P} 2 \mathrm{p}$ and $\mathrm{Pb} 4 \mathrm{f}$ overlapped with each other, we distinguish the molecules by the $\mathrm{O} 1 \mathrm{~s}$ peaks. The obviously increased intensity of $\mathrm{O}$ 1s in the XPS spectra (Fig. 1c) also showed evidence for the assembled organic molecules. The weak signal of $\mathrm{O} 1 \mathrm{~s}$ in the control $\mathrm{MAPbI}_{3}$ film might be attributed to the oxygen absorbed on the film from the environment. We further studied the effects of the interfacial treatment on the morphologies and crystallization of perovskites. As revealed in Fig. $\mathrm{S} 1, \dagger$ all the films treated with the bisphosphonic molecules showed dense configuration with no pinholes and analogical grain size. The roughness was around $4.21 \mathrm{~nm}$ to $5.53 \mathrm{~nm}$ for the surface-treated perovskite films, similar to the bare perovskite film, $5.67 \mathrm{~nm}$. XRD patterns in Fig. 1d revealed fine crystallinity of perovskites both with and without the interfacial treatment, which proved that the introduction of alkyl phosphoric molecules did not break the lattice structure of $\mathrm{MAPbI}_{3}$. Moreover, the optical absorption of 
(a)
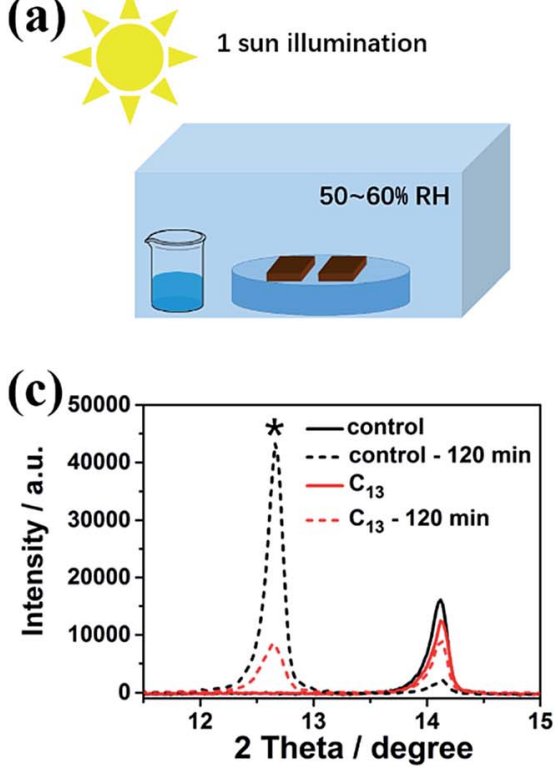
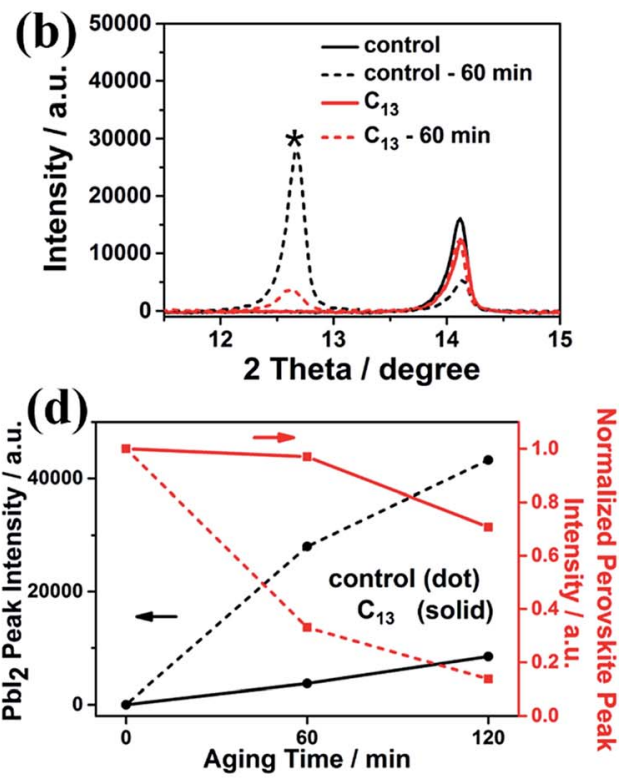

Fig. 2 (a) The scheme of the aging conditions under light and moisture. XRD spectra of the bare perovskite films and the $\mathrm{C}_{13}$-treated perovskite film after aged for (b) $60 \mathrm{~min}$ and (c) $120 \mathrm{~min}$. (d) The peak intensity of $\mathrm{Pbl}_{2}$ at $12.7^{\circ}$ and the normalized intensity peak of perovskite (110) plane at $14.1^{\circ}$ with the increase of aging time.

perovskite films in Fig. 1e showed no obvious change after the interfacial treatment. Therefore, we concluded that this interface engineering had negligible effect on the inner lattice structure and remained the outstanding absorption of the bulk perovskite films.

The synthesized alkyl bisphosphonic molecules were accepted with good hydrophobicity due to the steric effects and the lower polarity of the large alkyl groups, ${ }^{40}$ which was supposed to hinder moisture absorption and infiltration into the inner perovskite film. We tested the contact angle of water on the perovskite films with and without interfacial treatment to give direct evidence for the hydrophobicity change of the $\mathrm{MAPbI}_{3}$ film surface. To ensure the reliability, we selected five points with different location on each film. As shown in Fig. S2, $\dagger$ the average contact angle increased from $69.9^{\circ}$ to around $74^{\circ}$ after the surface treatment, indicating improved hydrophobicity of the perovskite film surface. To further study the stability of perovskite films before and after the surface treatment, we transferred the films into a sealed transparent box with $50-60 \% \mathrm{RH}$ under continuous AM1.5G illumination. As shown in Fig. S3, $\dagger$ the interfacial treatment with all the alkyl bisphosphonic molecules effectively retarded the degradation process compared with the bare $\mathrm{MAPbI}_{3}$ film, which manifested the efficacy of such hydrophobic bisphosphonic molecules in stability improvement. We compared the amplified XRD spectra of the bare $\mathrm{MAPbI}_{3}$ film and $\mathrm{C}_{13}$-treated film in Fig. 2. The $\mathrm{PbI}_{2}$ peak at $12.7^{\circ}$ in the XRD patterns of the bare $\mathrm{MAPbI}_{3}$ film increased greatly with the aging time, and the peak intensity of perovskite (110) plane decayed fast to $14 \%$ of its initial value under the impact of moisture and light (Fig. 2d). On the contrary, for the $\mathrm{C}_{13}$-treated film, the degradation into $\mathrm{PbI}_{2}$ was effectively hindered, with appearance of only a small $\mathrm{PbI}_{2}$ peak at $12.7^{\circ}$. The intensity of perovskite (110) peak remained $70 \%$ of the initial value after 120 min, indicating that the treated films possessed enhanced resistance against moisture and light. XPS spectra were also collected to further analyze the detailed degradation byproducts. As illustrated in Fig. 3a and b, the main peak of $\mathrm{Pb} 4 \mathrm{f}$ spectra at $138.3 \mathrm{eV}$ was corresponding to the $\mathrm{Pb}-\mathrm{I}$ binding energy in perovskite. ${ }^{41}$ When exposed to light and moisture, two additional peaks at $139.1 \mathrm{eV}$ and $136.5 \mathrm{eV}$ appeared in the bare perovskite film, attributed to the binding energy of $\mathrm{Pb}-\mathrm{I}$ bond in $\mathrm{PbI}_{2}$ and $\mathrm{Pb}^{0}$, respectively (Fig. 3c and d). ${ }^{42,43}$ It was reported that the loss of halide and methylammonium ions from the crystal could result in under-coordinated $\mathrm{Pb}$ atoms both on the crystal surface and at the grain boundaries. ${ }^{44}$ Here, the

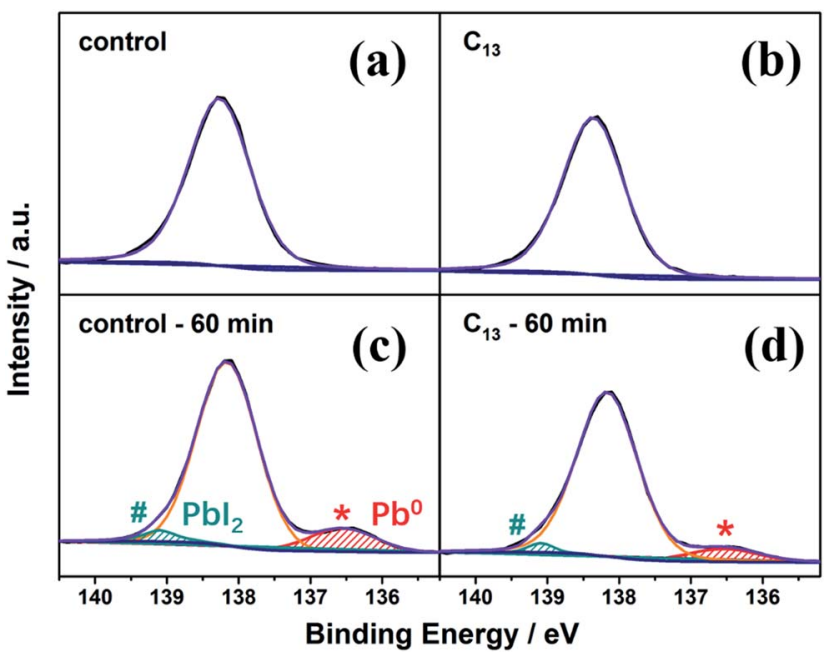

Fig. $3 \mathrm{~Pb} 4 \mathrm{f} X \mathrm{PS}$ spectra of the bare perovskite film ( $a$ and $\mathrm{c}$ ) and the $\mathrm{C}_{13}$-treated perovskite film ( $\mathrm{b}$ and $\mathrm{d}$ ) before and after aged in $50-60 \%$ $\mathrm{RH}$ atmosphere under AM1.5G illumination. 
presence of a substantial amount of $\mathrm{Pb}^{0}$ in the bare $\mathrm{MAPbI}_{3}$ film was supposed to be attributed to highly unsaturated $\mathrm{Pb}$ atoms, which were induced by the degradation under light, oxygen and moisture atmosphere. For the surface treated films, except for the reduced $\mathrm{PbI}_{2}$ peak, which was consistent with XRD results, the $\mathrm{Pb}^{0}$ peak also decreased, giving further evidence for the subdued degradation after the interfacial treatment with alkyl bisphosphonic molecules.

To verify the circumstance where the water molecules were surrounded by the amine ligands, the Bohn-Oppenheimer molecular dynamics (BOMD) simulations in Fig. 4 were performed to detect how the trapped water molecules would affect the perovskite films in the presence of the ligands. Looking from the bird-view in Fig. 4c and d, it could be clearly observed that the hydroxyl and oxygen of the bisphosphonic group formed a ten-membered ring in both $\mathrm{C}_{9}$ and $\mathrm{C}_{13}$ attachment. The longest diameter was measured between the opposing oxygen and phosphorus on the ring, which was calculated to be $4.8 \AA$. Considering the van der Waals radii of the atoms, the effective cavity only showed the longest diameter of $1.3 \AA$, which was even smaller than the van der Waals radius of a single oxygen atom $(1.5 \AA)$. Therefore, the ten-membered ring on top of the surface could prevent the water and oxygen from infiltrating into the perovskite structure. Based on the experimental and theoretical studies, we concluded that the surface treatment with the alkyl bisphosphonic molecules successfully hindered the moisture's infiltration and reaction with perovskite, decreased the loss of halide and methylammonium ions under moisture and light, and finally lead to the enhanced stability. (a)

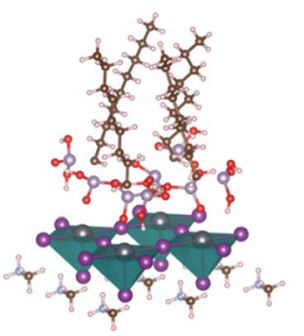

(b)

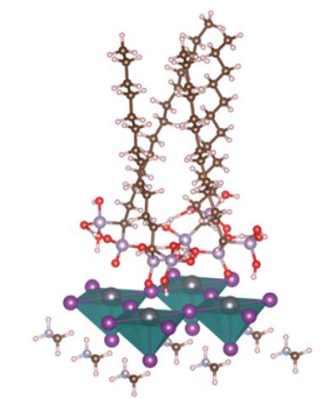

(c)

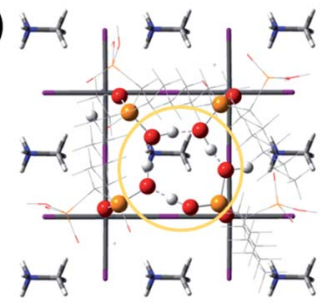

(d)

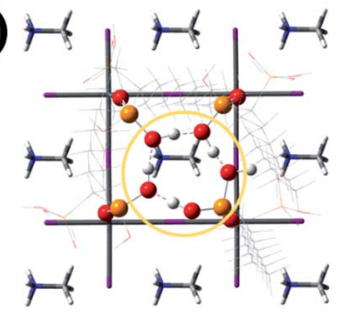

Fig. 4 (a) The 3D structure of the optimized perovskite cluster attached with four $\mathrm{C}_{9}$ molecules. (b) The 3D structure of the optimized perovskite cluster attached with four $\mathrm{C}_{13}$ molecules. (c) The bird-view of optimized geometry of four $\mathrm{C}_{9}$ molecules. (d) The bird-view of optimized geometry of four $\mathrm{C}_{13}$ molecules attached on the MAPbl 3 perovskite cluster. The ten-membered ring is highlighted by the yellow circle. The alkyl groups are indicated by the wireframes and the $\mathrm{MAPbl}_{3}$ is indicated by the tube for the visualization purpose.
The inverted p-i-n planar PVSCs were fabricated with $\mathrm{NiO}_{x}$ and $\mathrm{PC}_{61} \mathrm{BM}$ as the ETL and HTL, respectively, as illustrated in Fig. 5a. The energy levels of the fabricated device were also given in Fig. 5b, which matched well and ensured the efficient charge transport and collection in the devices. The $J-V$ curves and stabilized power output (SPO) curves of champion devices were shown in Fig. $5 \mathrm{c}$ and d, respectively. As seen in the corresponding performance parameters in Table $\mathrm{S} 1, \uparrow J_{\mathrm{sc}}, V_{\mathrm{oc}}$ and $\mathrm{FF}$ were all improved by the interfacial engineering. $\mathrm{C}_{13}$-treated devices showed the best performance, leading to an increase of PCE from $16.0 \%$ to $18.1 \%$. The corresponding SPO was also improved from $15.4 \%$ to $17.8 \%$. To note, the $\mathrm{PC}_{61} \mathrm{BM}$ deposition process might wash the phosphoric molecules away to some extent. However, the improved performance verified the efficient passivation by the interfacial engineering. We further collected statistic performance data of 5 batches devices, with 30 pixels for each condition (the control, the analogues treated with the four bisphosphonic molecules) to check the reliability of the surface treatment. As shown in Table 1, the average PCE of devices treated with alkyl bisphosphonic molecules markedly rose to over $17 \%$ compared to the control, and all the devices showed good reproducibility.

To rationally explain the performance improvement, the charge transfer at the perovskite/ $\mathrm{PC}_{61} \mathrm{BM}$ interface was further studied by the time-resolved PL spectra. As shown in Fig. S4, $\uparrow$ the charge transfer was accelerated by the interfacial treatment with the alkyl bisphosphonic molecules, with the lifetime decreased from $7.64 \mathrm{~ns}$ to $2.25 \mathrm{~ns}$. As electron donors, the bisphosphonic groups could provide lone pair electrons of $\mathrm{P}$ atoms, which could passivate the electric trap states at the perovskite/ $\mathrm{PC}_{61} \mathrm{BM}$ interface, reduce the recombination and facilitate the charge transfer. ${ }^{44,45}$ Moreover, since the long alkyl chains were insulating, the effect of the interfacial treatment on the surface conductivity of perovskite films was further studied by c-AFM (Fig. S5†). With the increase of the alkyl chain length, the surface conductivity of perovskite films showed an uniform decline tendency across the whole mapping area, which also indicated the uniform distribution of interfacial modification molecules on the perovskite film. Combining the surface passivation effects of bisphosphonic groups and conductivity variation induced by the long alkyl chain, devices with $\mathrm{C}_{13}$ treatment provided the best photovoltaic performance with the average PCE over $17 \%$, indicating sufficient performance improvement by the interfacial engineering with the bisphosphonic molecules.

We exposed as-fabricated devices to the $50-60 \% \mathrm{RH}$ atmosphere and continuous AM1.5G illumination to further investigate the stability variation. The PCE was averaged from 5 devices for each condition. As illustrated in Fig. S6, $\uparrow$ the interfacial treatment markedly diminished the PCE decay under moisture and light. Fig. 6a showed that the control device totally died while the devices treated with $\mathrm{C}_{13}$ remained over $70 \%$ of the initial PCE. We also studied the devices stability under ambient dark condition (20$30 \% \mathrm{RH})$. As illustrated in Fig. 6b, the unencapsulated devices with the interfacial treatment almost showed no degradation after exposed to ambient air for $300 \mathrm{~h}$, while the control device only remained less than $50 \%$ of its initial PCE. Therefore, the interfacial engineering with hydrophobic alkyl bisphosphonic molecules 
(a)

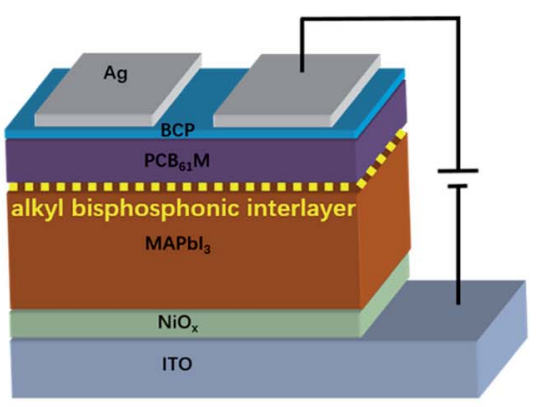

(c)

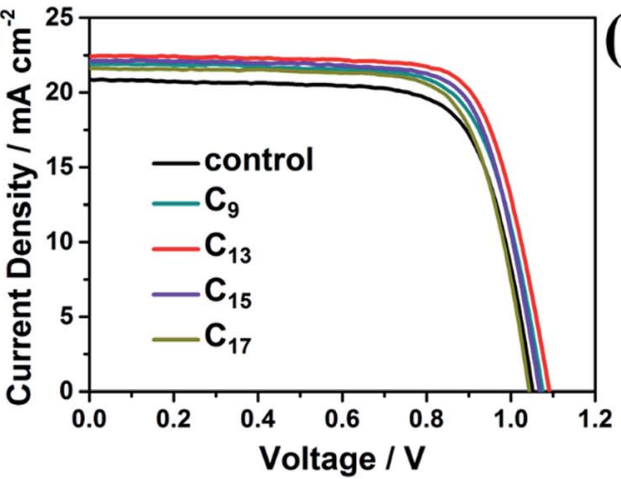

(b)

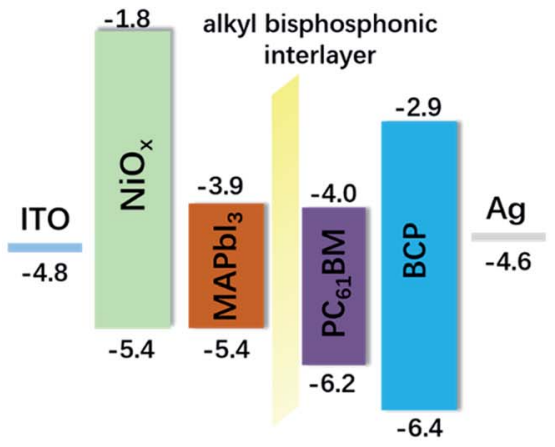

(d)

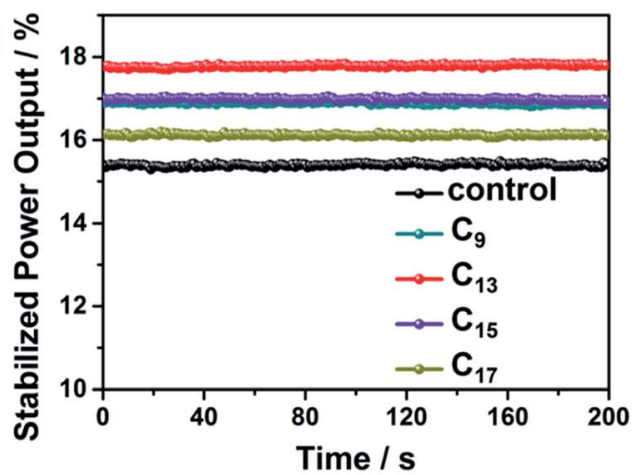

Fig. 5 (a) The scheme of the inverted PVSCs structure. (b) The energy level diagram of the inverted PVSCs. (c) J-V curves of the best performed devices $\mathrm{w} / \mathrm{o}$ the interfacial treatment. (d) The corresponding stabilized power output of the best performed devices. The $J-V$ characterization was measured with a scan rate of $100 \mathrm{mV} \mathrm{s}^{-1}$ under AM1.5G illumination.

Table 1 Statistic photovoltaic performance data of the inverted PVSCs

\begin{tabular}{lllll}
\hline & $J_{\mathrm{sc}} / \mathrm{mA} \mathrm{cm}^{-2}$ & $V_{\text {oc }} / \mathrm{V}$ & $\mathrm{FF} / \%$ & $\mathrm{PCE} / \%$ \\
\hline Control & $20.1 \pm 0.8$ & $1.04 \pm 0.01$ & $72.1 \pm 1.1$ & $15.1 \pm 1.0$ \\
$\mathrm{C}_{9}$ & $20.9 \pm 1.0$ & $1.05 \pm 0.02$ & $72.1 \pm 0.9$ & $15.8 \pm 1.3$ \\
$\mathrm{C}_{13}$ & $21.9 \pm 0.6$ & $1.07 \pm 0.01$ & $73.3 \pm 1.3$ & $17.2 \pm 0.9$ \\
$\mathrm{C}_{15}$ & $21.2 \pm 0.9$ & $1.05 \pm 0.01$ & $72.6 \pm 1.6$ & $16.2 \pm 1.2$ \\
$\mathrm{C}_{17}$ & $20.5 \pm 1.1$ & $1.04 \pm 0.01$ & $72.8 \pm 1.1$ & $15.5 \pm 1.2$
\end{tabular}

was proved to remarkably hinder the degradation under the continuous exposure to light and moisture, revealing the effectivity of interfacial treatment in moisture and light-stability improvement for high-efficiency inverted PVSCs.

\section{Conclusions}

In summary, we have demonstrated an efficient interfacial engineering by alkyl bisphosphonic molecules on top of perovskite films, which significantly improved the robustness of PVSCs under light and moisture, meanwhile enabling a PCE of $18.1 \%$ for the inverted PVSCs. The simultaneous enhancement of stability and efficiency was achieved through: (i) the hydrophobicity of the alkyl chains, (ii) the steric arrangement of the bisphosphonic molecules, which formed a ten-membered ring with a diameter smaller than the van der Waals radius of a single oxygen atom, (iii) the passivated trap states by lone pair electrons from the bisphosphonic groups. The experimental and theoretical results elucidated the molecular passivation principles to achieve
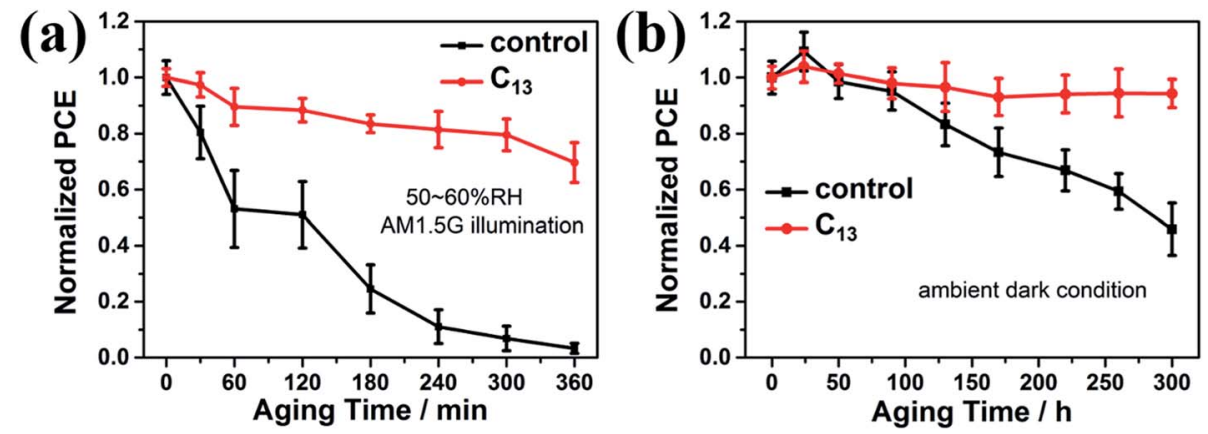

Fig. 6 (a) The stability test for the inverted PVSCs w/o the interfacial treatment in $50-60 \% \mathrm{RH}$ atmosphere under AM1.5G illumination. (b) The moisture stability test for the inverted PVSCs w/o the interfacial treatment in ambient (20-30\% RH) dark condition. All devices were unencapsulated. 
simultaneous efficiency and stability improvement, which is critical for advancing the material optimization processes toward practical applications of PVSCs.

\section{Conflicts of interest}

There are no conflicts to declare.

\section{Acknowledgements}

This work was funded by the National Natural Science Foundation of China under Grant No. 51273104 and 91433205. This work was facilitated though the use of advanced computational, storage, and networking infrastructure provided by the Hyak supercomputer system and funded by the STF at the University of Washington. Theoretical research is supported by the National Science Foundation (CHE-1565520 and CHE-1464497 to X. L.).

\section{References}

1 M. A. Green, A. Ho-Baillie and H. J. Snaith, Nat. Photonics, 2014, 8, 506-514.

2 N. J. Jeon, J. H. Noh, Y. C. Kim, W. S. Yang, S. Ryu and S. I. Seok, Nat. Mater., 2014, 13, 897-903.

3 J. Burschka, N. Pellet, S.-J. Moon, R. Humphry-Baker, P. Gao, M. K. Nazeeruddin and M. Gratzel, Nature, 2013, 499, 316319.

4 M. Grätzel, Acc. Chem. Res., 2017, 50, 487-491.

5 M. Gratzel, Nat. Mater., 2014, 13, 838-842.

6 G. Niu, X. Guo and L. Wang, J. Mater. Chem. A, 2015, 3, 89708980.

7 T. Leijtens, G. E. Eperon, N. K. Noel, S. N. Habisreutinger, A. Petrozza and H. J. Snaith, Adv. Energy Mater., 2015, 5, 1500963.

8 T. A. Berhe, W.-N. Su, C.-H. Chen, C.-J. Pan, J.-H. Cheng, H.-M. Chen, M.-C. Tsai, L.-Y. Chen, A. A. Dubale and B.-J. Hwang, Energy Environ. Sci., 2016, 9, 323-356.

9 Y. Rong, L. Liu, A. Mei, X. Li and H. Han, Adv. Energy Mater., 2015, 5, 1501066.

10 G. Niu, W. Li, F. Meng, L. Wang, H. Dong and Y. Qiu, J. Mater. Chem. A, 2014, 2, 705-710.

11 J. A. Christians, P. A. Miranda Herrera and P. V. Kamat, J. Am. Chem. Soc., 2015, 137, 1530-1538.

12 E. Mosconi, J. M. Azpiroz and F. De Angelis, Chem. Mater., 2015, 27, 4885-4892.

13 D. Bryant, N. Aristidou, S. Pont, I. Sanchez-Molina, T. Chotchunangatchaval, S. Wheeler, J. R. Durrant and S. A. Haque, Energy Environ. Sci., 2016, 9, 1655-1660.

14 J. M. Frost, K. T. Butler, F. Brivio, C. H. Hendon, M. van Schilfgaarde and A. Walsh, Nano Lett., 2014, 14, 2584-2590.

15 N. Aristidou, I. Sanchez-Molina, T. Chotchuangchutchaval, M. Brown, L. Martinez, T. Rath and S. A. Haque, Angew. Chem., Int. Ed., 2015, 54, 8208-8212.

16 W.-C. Lin, H.-Y. Chang, K. Abbasi, J.-J. Shyue and C. Burda, Adv. Mater. Interfaces, 2017, 4, 1600673.
17 S. N. Habisreutinger, D. P. McMeekin, H. J. Snaith and R. J. Nicholas, APL Mater., 2016, 4, 091503.

18 W. Ke, G. Fang, Q. Liu, L. Xiong, P. Qin, H. Tao, J. Wang, H. Lei, B. Li, J. Wan, G. Yang and Y. Yan, J. Am. Chem. Soc., 2015, 137, 6730-6733.

19 Z. Zhu, Y. Bai, X. Liu, C.-C. Chueh, S. Yang and A. K. Y. Jen, Adv. Mater., 2016, 28, 6478-6484.

20 J. You, L. Meng, T.-B. Song, T.-F. Guo, Y. Yang, W.-H. Chang, Z. Hong, H. Chen, H. Zhou, Q. Chen, Y. Liu, N. De Marco and Y. Yang, Nat. Nanotechnol., 2016, 11, 75-81.

21 L. Q. Zhang, X. W. Zhang, Z. G. Yin, Q. Jiang, X. Liu, J. H. Meng, Y. J. Zhao and H. L. Wang, J. Mater. Chem. A, 2015, 3, 12133-12138.

22 S. Bai, Z. Wu, X. Wu, Y. Jin, N. Zhao, Z. Chen, Q. Mei, X. Wang, Z. Ye, T. Song, R. Liu, S.-t. Lee and B. Sun, Nano Res., 2014, 7, 1749-1758.

23 A. Bera, A. D. Sheikh, M. A. Haque, R. Bose, E. Alarousu, O. F. Mohammed and T. Wu, ACS Appl. Mater. Interfaces, 2015, 7, 28404-28411.

24 S. S. Shin, W. S. Yang, J. H. Noh, J. H. Suk, N. J. Jeon, J. H. Park, J. S. Kim, W. M. Seong and S. I. Seok, Nat. Commun., 2015, 6, 7410.

25 L. Zhu, Z. Shao, J. Ye, X. Zhang, X. Pan and S. Dai, Chem. Commun., 2016, 52, 970-973.

26 W. Chen, Y. Wu, Y. Yue, J. Liu, W. Zhang, X. Yang, H. Chen, E. Bi, I. Ashraful, M. Grätzel and L. Han, Science, 2015, 350, 944-948.

27 D. H. Cao, C. C. Stoumpos, O. K. Farha, J. T. Hupp and M. G. Kanatzidis, J. Am. Chem. Soc., 2015, 137, 7843-7850.

28 H. Tsai, W. Nie, J.-C. Blancon, C. C. Stoumpos, R. Asadpour, B. Harutyunyan, A. J. Neukirch, R. Verduzco, J. J. Crochet, S. Tretiak, L. Pedesseau, J. Even, M. A. Alam, G. Gupta, J. Lou, P. M. Ajayan, M. J. Bedzyk, M. G. Kanatzidis and A. D. Mohite, Nature, 2016, 536, 312-316.

29 I. C. Smith, E. T. Hoke, D. Solis-Ibarra, M. D. McGehee and H. I. Karunadasa, Angew. Chem., 2014, 126, 11414-11417.

30 K. Yao, X. Wang, Y.-x. Xu, F. Li and L. Zhou, Chem. Mater., 2016, 28, 3131-3138.

31 J. Zhang, Z. Hu, L. Huang, G. Yue, J. Liu, X. Lu, Z. Hu, M. Shang, L. Han and Y. Zhu, Chem. Commun., 2015, 51, 7047-7050.

32 H. Si, Q. Liao, Z. Zhang, Y. Li, X. Yang, G. Zhang, Z. Kang and Y. Zhang, Nano Energy, 2016, 22, 223-231.

33 M. Kot, C. Das, Z. Wang, K. Henkel, Z. Rouissi, K. Wojciechowski, H. J. Snaith and D. Schmeisser, ChemSusChem, 2016, 9, 3401-3406.

34 X. Dong, X. Fang, M. Lv, B. Lin, S. Zhang, J. Ding and N. Yuan, J. Mater. Chem. A, 2015, 3, 5360-5367.

35 D. Koushik, W. J. H. Verhees, Y. Kuang, S. Veenstra, D. Zhang, M. A. Verheijen, M. Creatore and R. E. I. Schropp, Energy Environ. Sci., 2017, 10, 91-100.

36 G. Niu, W. Li, F. Meng, L. Wang, H. Dong and Y. Qiu, J. Mater. Chem. A, 2014, 2, 705-710.

37 T. Leijtens, G. E. Eperon, S. Pathak, A. Abate, M. M. Lee and H. J. Snaith, Nat. Commun., 2013, 4, 2885.

38 Z. Yang, C.-C. Chueh, P.-W. Liang, M. Crump, F. Lin, Z. Zhu and A. K. Y. Jen, Nano Energy, 2016, 22, 328-337. 
39 Z. Bin, J. Li, L. Wang and L. Duan, Energy Environ. Sci., 2016, 9, 3424-3428.

40 S. Yang, Y. Wang, P. Liu, Y.-B. Cheng, H. J. Zhao and H. G. Yang, Nat. Energy, 2016, 1, 15016.

41 B. Conings, L. Baeten, C. De Dobbelaere, J. D'Haen, J. Manca and H.-G. Boyen, Adv. Mater., 2014, 26, 2041-2046.

42 S. R. Raga, M.-C. Jung, M. V. Lee, M. R. Leyden, Y. Kato and Y. Qi, Chem. Mater., 2015, 27, 1597-1603.
43 W. Li, J. Li, G. Niu and L. Wang, J. Mater. Chem. A, 2016, 4, 11688-11695.

44 N. K. Noel, A. Abate, S. D. Stranks, E. S. Parrott, V. M. Burlakov, A. Goriely and H. J. Snaith, ACS Nano, 2014, 8, 9815-9821.

45 D. W. deQuilettes, S. Koch, S. Burke, R. K. Paranji, A. J. Shropshire, M. E. Ziffer and D. S. Ginger, ACS Energy Lett., 2016, 1, 438-444. 